\title{
Thyroid Dysfunction in Patients with Liver Cirrhosis
}

\author{
Joeimon J L ${ }^{1}$,Mohanraj $\mathrm{K}^{2}$,Karthikeyan $\mathrm{R}^{2}$, \\ Rajkumar Solomon $\mathrm{T}^{3}$,Aravind $\mathrm{A}^{3}$, Caroline Selvi $\mathrm{K}^{3}$,Balamurali $\mathrm{R}^{4}$, Ramkumar \\ $\mathrm{G}^{4}$, Muthukumuran $\mathrm{K}^{4}$,Vaishnavipriyaa $\mathrm{C}^{4}$,Kavitha $\mathrm{S}^{4}$,Anand $\mathrm{A}^{4}$ \\ Department Of Digestive Health And Digeases (Ddhd), Kilpauk Medical College (Kmc), Chennai, Tamil Nadu, \\ India
}

\begin{abstract}
Aim: To estimate the prevalence of thyroid dysfunction in patients with liver cirrhosis.

Materials and Methods: Early morning fasting serum thyroid stimulating hormone (TSH), serum total free thyroxine (FT4) and free triiodothyronine (FT3) was measured by radioimmunoassays in 111 index patients with liver cirrhosis who did not have history of thyroid diseases. Study period wasoctober 2014 to december 2016. The age of study population was in the range of 25-60 years. Out of 111 patients, 80 were males and 31 were females.Out of 111 patients 64 had ethanol related liver disease, 21 had Hepatitis B positivity, 5 had hepatitis $C$ positivity, 5 had autoimmune hepatitis, 4 had wilson disease and 12 had crytogenic cirrhosis.

Out of the 111 patients, 28 belonged CTP A, 53 belonged CTP B and 30 belonged CTP C.

Results: Hypothyroidism was seen in 24 out of 111 patients (21.6\%). Only serum TSH increase was seen in 12 patients (10.8\%) and Serum TSH increase along with FT3 and FT4 decrease in 12 patients out of 111 patients (10.8\%). Of the 24 patients having hypothyroidism, 5 belonged to CTP A (out of 28 i.e 17.8\%), 11 belonged to CTP B (out of 53 i.e 20.7\%), and 8 belonged to CTP C (out of 30 i.e 26.6\%).Of the 24 patients having hypothyroidism, 16 (66.6\%) were those having ethanol related liver cirrhosis, 4 (16.6\%) have Hepatitis B positivity and $4(16.6 \%)$ have other causes of liver cirrhosis. Out of the 64 patients who had ethanol related liver cirrhosis 16 had hypothyroidism (25\%) and 4 out of 21 patients having Hepatitis $B$ related liver cirrhosis had hypothyroidism (19\%).

Conclusion: Abnormalities in circulating thyroid hormone concentrations i.e hypothyroidism is noted especially in those with ethanol related liver cirrhosis and it is associated with more advanced liver disease.

Keywords: Liver cirrhosis, Hypothyroidism, Ethanol related liver disease, Hepatitis B related liver disease, CTP score.

\section{Introduction}

The liver has an important role in thyroid hormone metabolism because it is the manufacturer of proteins that bind thyroid hormone, such as thyroxine-binding globulin (TBG), pre-albumin and albumin. It is also the major site of thyroid hormone peripheral metabolism and is involved in its conjugation, biliary excretion, oxidative deamination and the extrathyroidaldeiodination of thyroxine (T4) to triiodothyronine (T3) and to reverse T3. On the other hand, the level of thyroid hormones is also important to normal hepatic function and bilirubin metabolism, Namely: (i) liver damage secondary to the systemic effect of thyroid hormone excess or direct toxic effects and subclinical physiological effects of thyroid hormone on liver function; (ii) some patients with chronic liver diseases may have thyroiditis, hyperthyroidism or hypothyroidism through autoimmune mechanisms; (iii) alterations of thyroid hormone metabolism or tests secondary to liver disease, and (iv) liver or thyroid disorders related to the therapy of thyroid or liver disease.
\end{abstract}

Aim

- To estimate the prevalence of thyroid dysfunction in patients with liver cirrhosis.

- To assess the correlation between thyroid dysfunction and the severity of liver cirrhosis.

Study design:

\section{Materials And Methods}

Prospective cross sectional observational study

Inclusion criteria:

Patients with symptoms, signs, biochemical and radiological features of liver cirrhosis irrespective of cause.

Exclusion criteria:

Liver Disease patients on treatment already and with any complications like hepatic encephalopathy, spontaneous bacterial peritonitis, hepatorenal syndrome, gastrointestinal bleed and patients on thyroid medications already. 


\section{Informed Consent:}

Study groups were informed about the nature of the study and the selected participants were included in the study after getting written informed consent.

\section{Study period:}

Study period was October 2014 to December 2016.

\section{Study Methodology:}

Early morning fasting serum thyroid stimulating hormone (TSH), serum total free thyroxine (FT4) and free triiodothyronine (FT3) was measured by radioimmunoassays in 111 index patients with liver cirrhosis who did not have history of thyroid diseases.

The age of study population was in the range of 25-60 years. Out of 111 patients, 80 were males and 31 were females.Each patient's complete history was noted. Every patient was investigated after the completion of physical examination. Out of 111 patients, 64 had ethanol related liver disease, 21 had Hepatitis B positivity, 5 had hepatitis $\mathrm{C}$ positivity, 5 had autoimmune hepatitis, 4 had Wilson disease and 12 had cryptogenic cirrhosis.

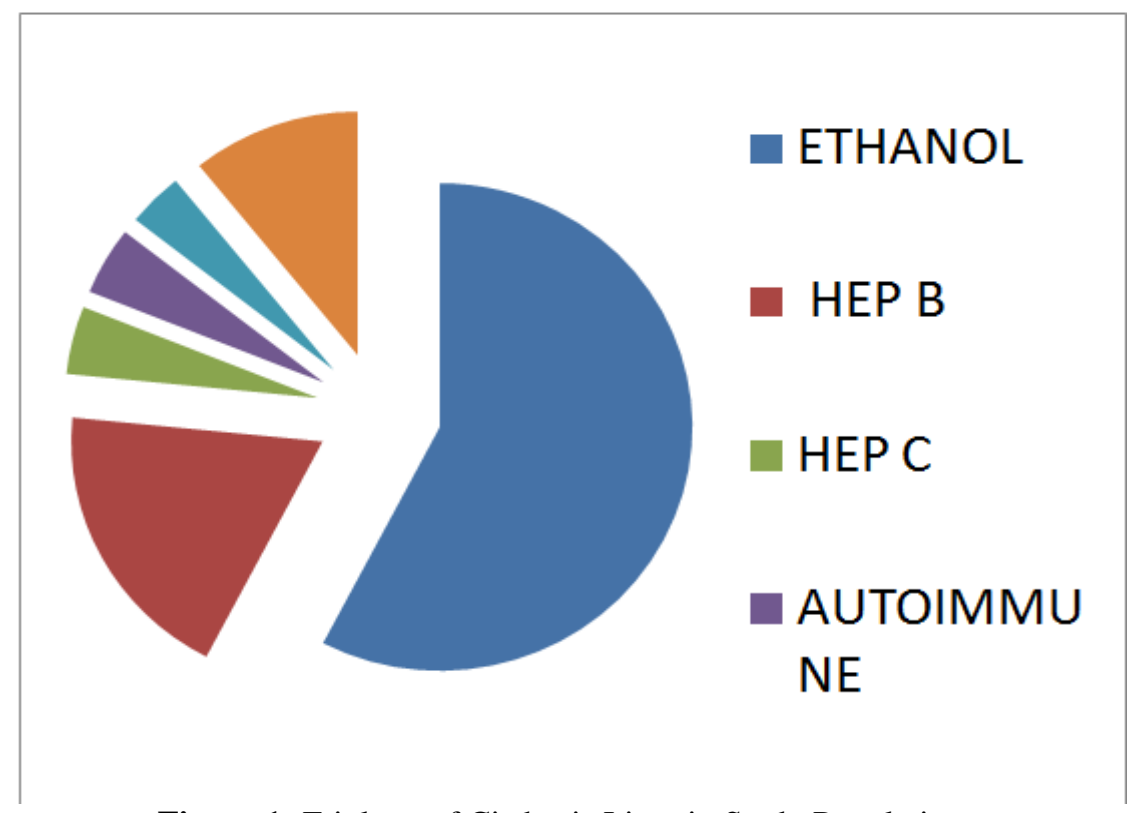

Figure 1: Etiology of Cirrhosis Liver in Study Population

Out of the 111 patients, 28 belonged CTP A, 53 belonged CTP B and 30 belonged CTP C.

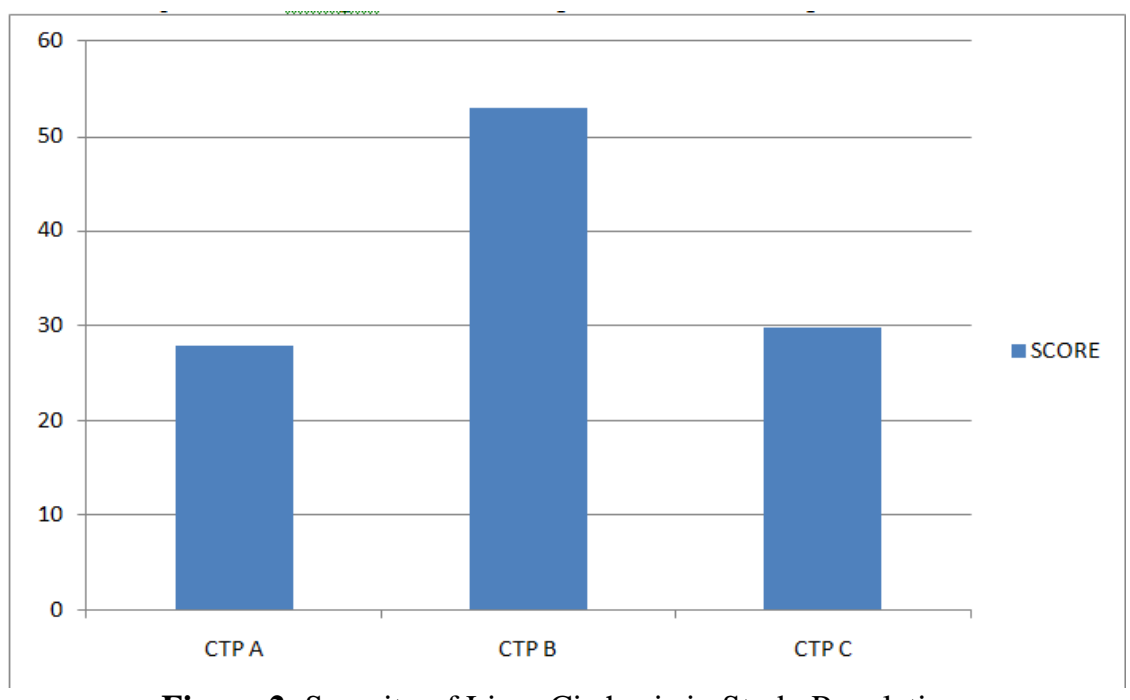

Figure 2: Severity of Liver Cirrhosis in Study Population 


\section{Results}

Hypothyroidism was seen in 24 out of 111 patients (21.6\%). Only serum TSH increase was seen in 12 patients $(10.8 \%)$ and Serum TSH increase along with FT3 and FT4 decrease in 12 patients out of 111 patients $(10.8 \%)$.

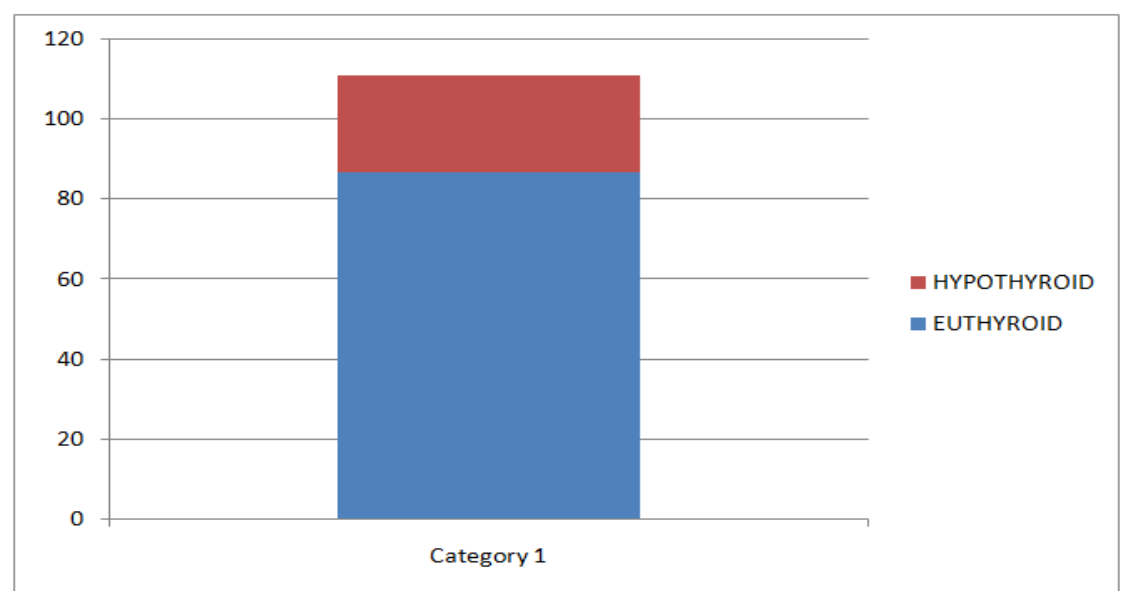

Figure 3: Prevalence of Hypothyroidism

Of the 24 patients having hypothyroidism, 5 belonged to CTP A (out of 28 i.e 17.8\%), 11 belonged to CTP B (out of 53 i.e 20.7\%), and 8 belonged to CTP C (out of 30 i.e 26.6\%).

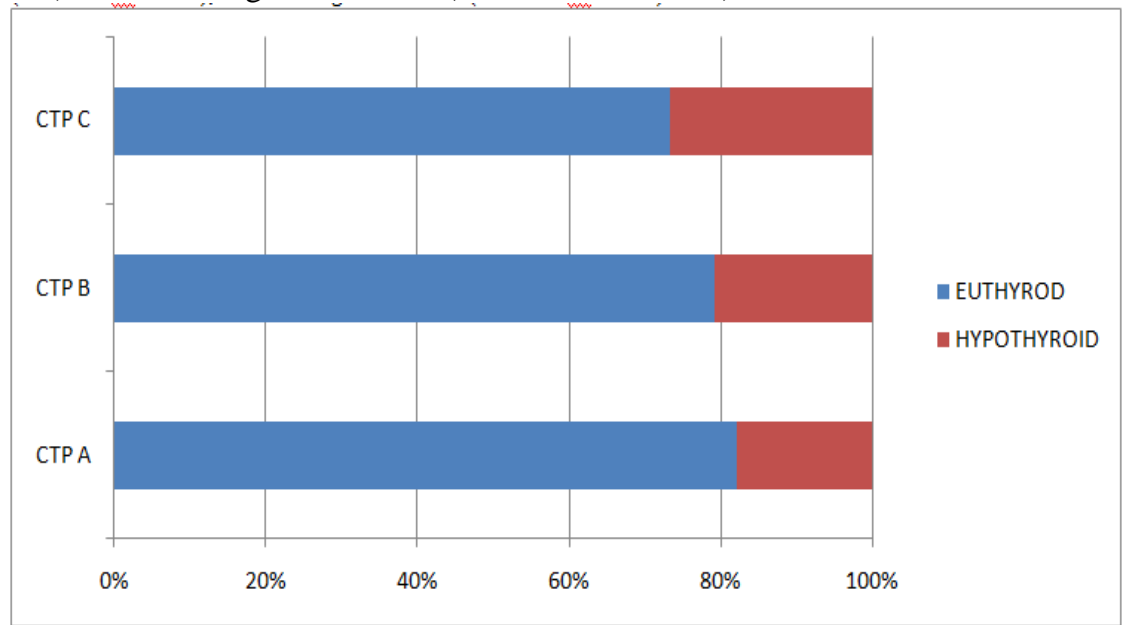

Figure 4: Prevalence of Hypothyroidism based on cirrhosis severity

Of the 24 patients having hypothyroidism, $16(66.6 \%)$ were those having ethanol related liver disease, 4 $(16.6 \%)$ have Hepatitis B positivity and $4(16.6 \%)$ have other causes of liver disease.

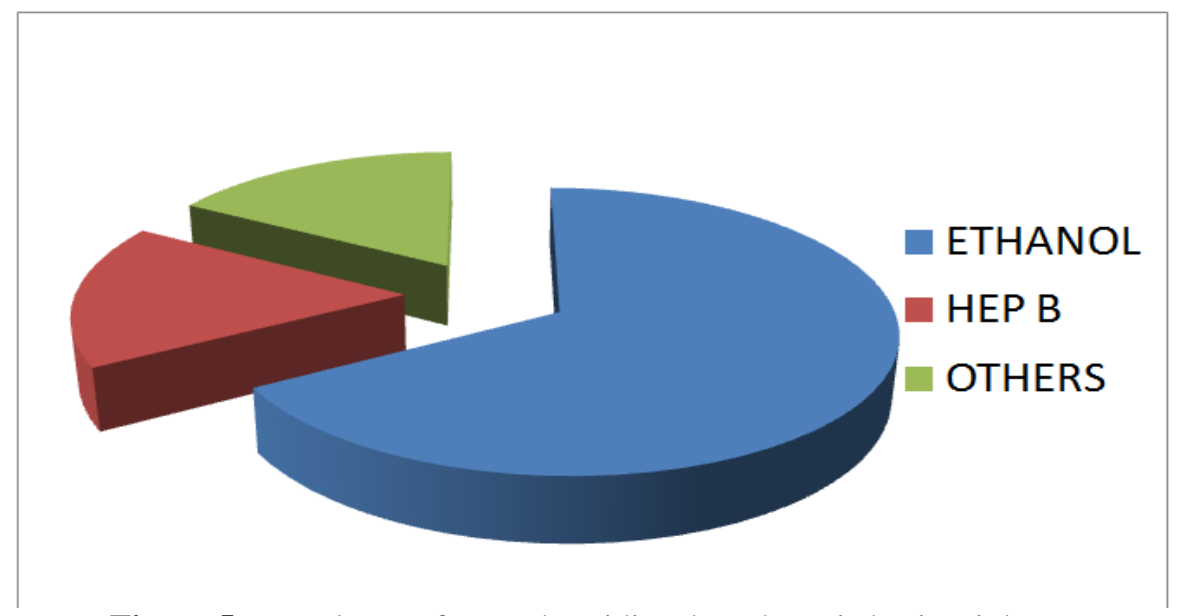

Figure 5: Prevalence of Hypothyroidism based on cirrhosis etiology 
Out of the 64 patients who had ethanol related liver cirrhosis 16 had hypothyroidism (25\%) and 4 out of 21 patients having Hepatitis B related liver cirrhosis had hypothyroidism (19\%).

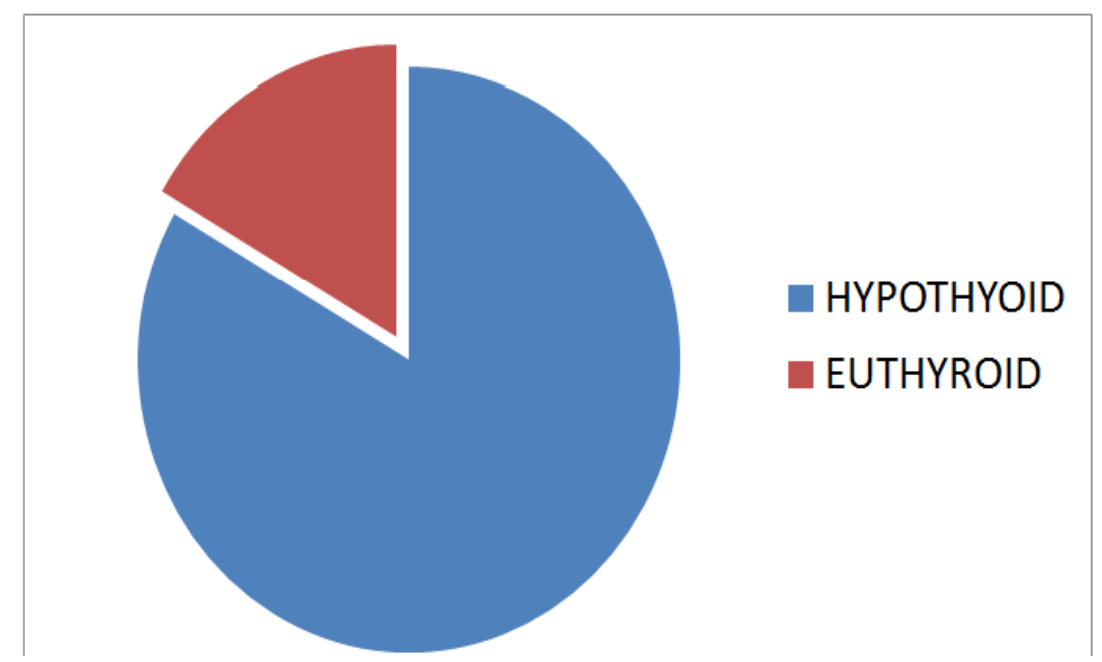

Figure 6: Prevalence of Hypothyroidism based on ethanol related cirrhosis

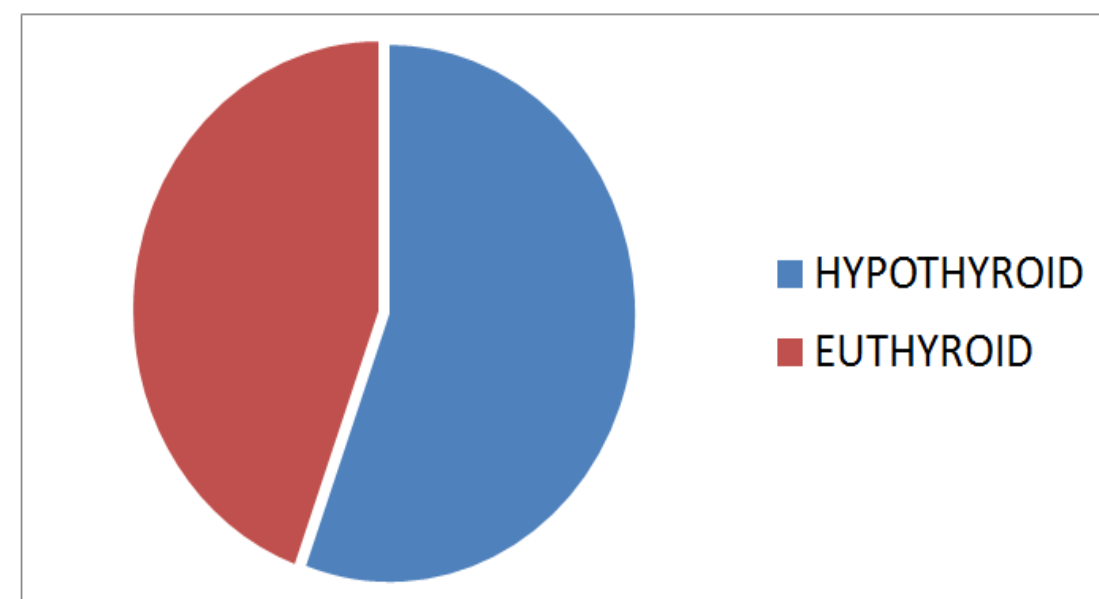

Figure 7: Prevalence of Hypothyroidism based on Hepatitis B related cirrhosis

\section{Discussion}

The liver has an important role in thyroid hormone metabolism and the level of thyroid hormones is also important to normal hepatic function and bilirubin metabolism.Besides the associations between thyroid and liver diseases of an autoimmune nature, such as that between primary biliary cirrhosis and hypothyroidism, thyroid diseases are frequently associated with liver injuries or biochemical test abnormalities. Liver diseases are also frequently associated with thyroid test abnormalities or dysfunctions, particularly elevation of thyroxine-binding globulin and thyroxine. According to Takahashi et al, The abnormalities of serum levels of thyroid hormones are frequently found in liver diseases. The pattern of abnormalities may be observed according to the type of disease and its severity. The estimation of free thyroid hormones level is important in liver dysfunction because of the changes of the binding protein in blood.Serum FT4 concentration is decreased only in cirrhosis of liver. It is observed that the decrease of serum FT4 concentration was noted in critical stages of liver diseases as in other diseases. Hence serum FT4 concentration is presumed to be a useful index for prognosis and has a different significance from serum FT3 level. Hepner and Walfish observed a significant inverse correlation between serum T3 value and the severity of liver dysfunction. A progressive fall in T3 concentration in cirrhosis of liver was shown as an indicator of poor prognosis. Hitomi et al studied on changes of thyroid hormones in various liver diseases which showed usefulness of free thyroid hormones as liver function test.

Hodgson et al studied the relationship between the thyroid gland and the liver. He observed that thyroxine and tri-iodothyronine hormones modulate hepatic function. The liver metabolizes the thyroid hormones and inturn regulates their systemic and endocrine effects. Thyroid dysfunction may affect liver 
function, liver disease inturn modulates thyroid hormone metabolism, and many systemic diseases affect both organs.

In our study as the severity of liver disease increases and the CTP score raises the prevalence of thyroid dysfunction increases. And this hypothyroidism may have an impact on the survival of the patients. Neglect of these facts may result in over of under diagnosis of associated liver or thyroid diseases and thereby cause errors in patient care.

It is suggested to measure free thyroxine (FT4) and thyroid-stimulating hormone (TSH) which are usually normal in euthyroid patients with liver disease, to rule out or rule in coexistent thyroid dysfunctions, and consider the possibility of thyroid dysfunctions in any patients with liver abnormalities.

\section{Conclusion}

- Abnormalities in circulating thyroid hormone concentrationsi.e hypothyroidism is noted in those with liver cirrhosis

- Hypothyroidism is noted especially in those with ethanol related cirrhosis liver

- Hypothyroidism is associated with more advanced liver disease

\section{Acqnowledments}

The authors are highly indebted to the Almighty God and the faculties of Department of Medical Gastroenterology, Department of Digestive Health and Diseases (DDHD), Kilpauk Medical College (KMC), Chennai, India for their support and expert opinion during formulation/ designing of the study on the basis of which this study was made possible.

\section{References}

[1]. The Liver in Systemic Disease, Fagiuoli S., Van Thiel D. H., 1993

[2]. Associations between diseases of the thyroid and the liver, Babb R. R., Am. J. Gastroenterol., 1984

[3]. Thyroid hormone homeostasis and the liver, Salata R., Klein I., LeveyG..Semin. Liver Dis., 1985

[4]. Changes in thyroid hormone concentration in liver disease Huang M. J., Liaw Y. F., J. Formosan Med. Assoc., 1989

[5]. Clinical associations between thyroid and liver diseases. Huang MJ , Liaw YF., JGGastroenterolHepatol. 1995 May-Jun;10(3):34450 .

[6]. Chopra IJ. Serum thyroid hormone levels in patients with liver disease. Arch Intern Med 1979; 139: 1117-20.

[7]. Orrego H, Israel Y, Blake J. Serum triiodothyronine and other clinical and laboratory indices of alcoholic liver disease. Ann Intern Med 1979; 91:13-6.

[8]. Walfish PG, Orrego H, Blake J. Thyroid hormones in alcoholic liver disease: effect of treatment with 6-propylthiouracil. Gastroenterology 1979; 76: 116-22.

[9]. Snitcher EJ, Mowat NAG, Ekins RP, Rees LH. Thyroid function and thyroid regulation in euthyroid men with cirrhosis of liver : evidence of multiple abnormalities. ClinEndocrinol 1977; 7: 453-61.

[10]. Thyroid function in early and late alcohol withdrawal: relationship with aggression, family history, and onset age of alcoholism. Ertugrulet a, Hasanbasri and Sehersofuoglu. Oxford university press on behalf of the medical council on alcohol. 